\title{
Loss of males from mixed-sex societies in termites
}

\author{
Toshihisa Yashiro ${ }^{1,2^{*}} \mathbb{D}$, Nathan Lo ${ }^{1}$, Kazuya Kobayashi² ${ }^{2}$, Tomonari Nozaki² ${ }^{\mathbb{D}}$, Taro Fuchikawa ${ }^{2} \mathbb{D}$, \\ Nobuaki Mizumoto ${ }^{2}$ (D) Yusuke Namba² and Kenji Matsuura ${ }^{2}$ (B)
}

\begin{abstract}
Background: Sexual reproduction is the norm in almost all animal species, and in many advanced animal societies, both males and females participate in social activities. To date, the complete loss of males from advanced social animal lineages has been reported only in ants and honey bees (Hymenoptera), whose workers are always female and whose males display no helping behaviors even in normal sexual species. Asexuality has not previously been observed in colonies of another major group of social insects, the termites, where the ubiquitous presence of both male and female workers and soldiers indicate that males play a critical role beyond that of reproduction.
\end{abstract}

Results: Here, we report asexual societies in a lineage of the termite Glyptotermes nakajimai. We investigated the composition of mature colonies from ten distinct populations in Japan, finding six asexual populations characterized by a lack of any males in the reproductive, soldier, and worker castes of their colonies, an absence of sperm in the spermathecae of their queens, and the development of unfertilized eggs at a level comparable to that for the development of fertilized eggs in sexual populations of this species. Phylogenetic analyses indicated a single evolutionary origin of the asexual populations, with divergence from sampled sexual populations occurring about 14 million years ago. Asexual colonies differ from sexual colonies in having a more uniform head size in their allfemale soldier caste, and fewer soldiers in proportion to other individuals, suggesting increased defensive efficiencies arising from uniform soldier morphology. Such efficiencies may have contributed to the persistence and spread of the asexual lineage. Cooperative colony foundation by multiple queens, the single-site nesting life history common to both the asexual and sexual lineages, and the occasional development of eggs without fertilization even in the sexual lineage are traits likely to have been present in the ancestors of the asexual lineage that may have facilitated the transition to asexuality.

Conclusions: Our findings demonstrate that completely asexual social lineages can evolve from mixed-sex termite societies, providing evidence that males are dispensable for the maintenance of advanced animal societies in which they previously played an active social role.

Keywords: All-female asexual societies, Asexual social lineages, Thelytokous parthenogenesis, Sexual reproduction, Advanced social animals, Social insects

\section{Background}

Sexual reproduction is nearly ubiquitous in nature [1] and is thought to provide key selective advantages [2]. Sexual reproduction enables gene pools to be constantly mixed and generates new combinations of genes, facilitating adaptation to changing environments [3].

\footnotetext{
* Correspondence: toshihisa.yashiro@sydney.edu.au

${ }^{1}$ School of Life and Environmental Sciences, Edgeworth David Building A11, University of Sydney, Sydney, NSW 2006, Australia

${ }^{2}$ Laboratory of Insect Ecology, Graduate School of Agriculture, Kyoto University, Kyoto 606-8502, Japan
}

Nevertheless, some organismal lineages can exist without sex $[1,4]$. Although asexual lineages occur sporadically in diverse animal taxa [1,4], their sexual ancestors are often thought to have been pre-adapted genetically and/or ecologically for persistence following the loss of sexual reproduction $[5,6]$.

In the context of social insect colonies, the genetic diversity resulting from sexual reproduction is thought to provide multiple benefits, including enhanced disease resistance and resilient division of labour [7]. Despite these benefits, sexual reproduction is bypassed in advanced 
societies of some hymenopteran insects (ants and honeybees), leading to completely asexual lineages $[8,9]$. Indeed, hymenopteran social insect species are thought to be pre-adapted to thelytokous parthenogenesis (asexual production of diploid females), owing to their haplodiploid sex determination system, where males are produced via arrhenotokous parthenogenesis (asexual production of haploid males) [5, 9]. Furthermore, among hymenopteran social insects, including normal sexual species, workers are always female; males display no helping behavior (i.e., societies are essentially all-female). This is thought to be an outcome of sex-specific pre-adaptations for helping in the solitary ancestors of social species [10].

In contrast to hymenopteran social insects, termites are diploid and typically have an XY sex determination system [11]. Their colonies commonly comprise both male and female reproductives, workers, and soldiers [12]. For example, although there is a variety of caste developmental pathways in termites, males and females of dry-wood termites (family Kalotermitidae) follow the same developmental pathway, through which the worker caste has totipotency to differentiate into other castes, including those of reproductives and soldiers [13, 14] (Fig. 1). The complete loss of males from termite lineages would therefore result not only in the loss of sexual reproduction, but also the loss of sex differences in the workforce (often due to size dimorphism) [15]. Termite parthenogenesis is expected to be thelytokous rather than arrhenotokous because of the XY sex determination system found in most species [16]. Although thelytokous parthenogenesis has been reported in some termites, this is restricted to the production of neotenic (secondary) reproductives in nature $[16,17]$ (but see also $[18,19]$ for the ability of parthenogenetic colony foundation of these "asexual queen succession (AQS)" termites in the laboratory). Importantly, both male and female workers and soldiers continue to be produced sexually in natural AQS colonies [16, 17], indicating that males play a critical role beyond that of reproduction in termite societies. To date, no termite colony in the field has ever been found to completely lack males.

The dry-wood termite Glyptotermes nakajimai Morimoto (Isoptera: Kalotermitidae) is widely distributed in coastal areas of the mainland (Honshu, Shikoku, and Kyushu) and small island regions (Amami-Oshima Island, Okinawa Islands, and Ogasawara Islands) of Japan $[20,21]$. Previous studies of this species showed that their colonies contain both males and females [22]. However, our preliminary examinations indicated that colonies in some populations are exclusively female, including both worker and reproductive castes. We therefore hypothesized the evolutionary loss of males from representatives of this species.
We tested this hypothesis in a series of morphological, molecular, and cytological analyses, including sexing of individual termites and examination of sperm storage among egg-laying queens. Based on phylogenetic evidence of a single origin of asexuality, we further explored the evolution of the asexual lineage through comparative studies of asexual and sexual G. nakajimai.

\section{Results and discussion \\ Glyptotermes nakajimai contains multiple asexual populations}

To confirm the presence of asexual populations of $G$. nakajimai, we investigated the colony composition of 74 mature colonies from ten populations. Colonies contained multiple reproductives and up to 2300 non-reproductive members (soldiers, workers, nymphs, alates, and young instars; see Fig. 1). In lower termites, including the family Kalotermitidae, individuals (except young instars) usually exhibit sexual dimorphism in their external morphology, such as the female-specific character of having an elongated seventh sternite [23, 24] (but see [25]). Dissections revealed that our sexing of $G$. nakajimai individuals on the basis of the configuration of the caudal sternites was $100 \%$ accurate in reproductives $(n=30)$, soldiers $(n=30)$, and workers $(n=60)$ (Additional file 1: Figure S1), confirming that the sex of these castes of G. nakajimai can be easily determined from sternite morphology. In the Shikoku and Kyushu populations, all reproductives $(n=194)$, soldiers $(n=$ $316)$, and workers $(n=3700)$ collected from 37 colonies were female (Fig. 2a and Table 1). In contrast, all 37 colonies collected from the other populations (Honshu, Amami-Oshima Island, Okinawa Island, and Ogasawara Islands) contained both male and female reproductives (females $/[$ females + males $]=0.53 \pm 0.02$ SEM $[n=548]$ ), soldiers (females/[females + males] $=0.42 \pm 0.03 \mathrm{SEM}[n$ $=713]$ ), and workers (females $/[$ females + males $]=0.49 \pm$ 0.01 SEM $[n=3700]$ ) (Fig. 2a and Table 2).

To rule out the possibility that cryptic males exist in the all-female populations (Shikoku and Kyushu), we examined sperm storage among egg-laying queens by observing the spermathecae (sperm storage organs) stained by propidium iodide. Spermathecae of all examined queens $(n=12)$ in the all-female populations of G. nakajimai were empty, whereas those of all examined queens $(n=12)$ in the other mixed-sex populations were filled with sperm $(P<0.0001$, Fisher's exact probability test) (Fig. 2a). This is, to our knowledge, the first demonstration of queens in natural colonies that lack sperm in their spermathecae. In all the cases, we could find in the literature every termite queen in natural colonies that has been checked has had sperm in her spermatheca, regardless of the presence or absence of the kings (e.g., [26, 27]). 


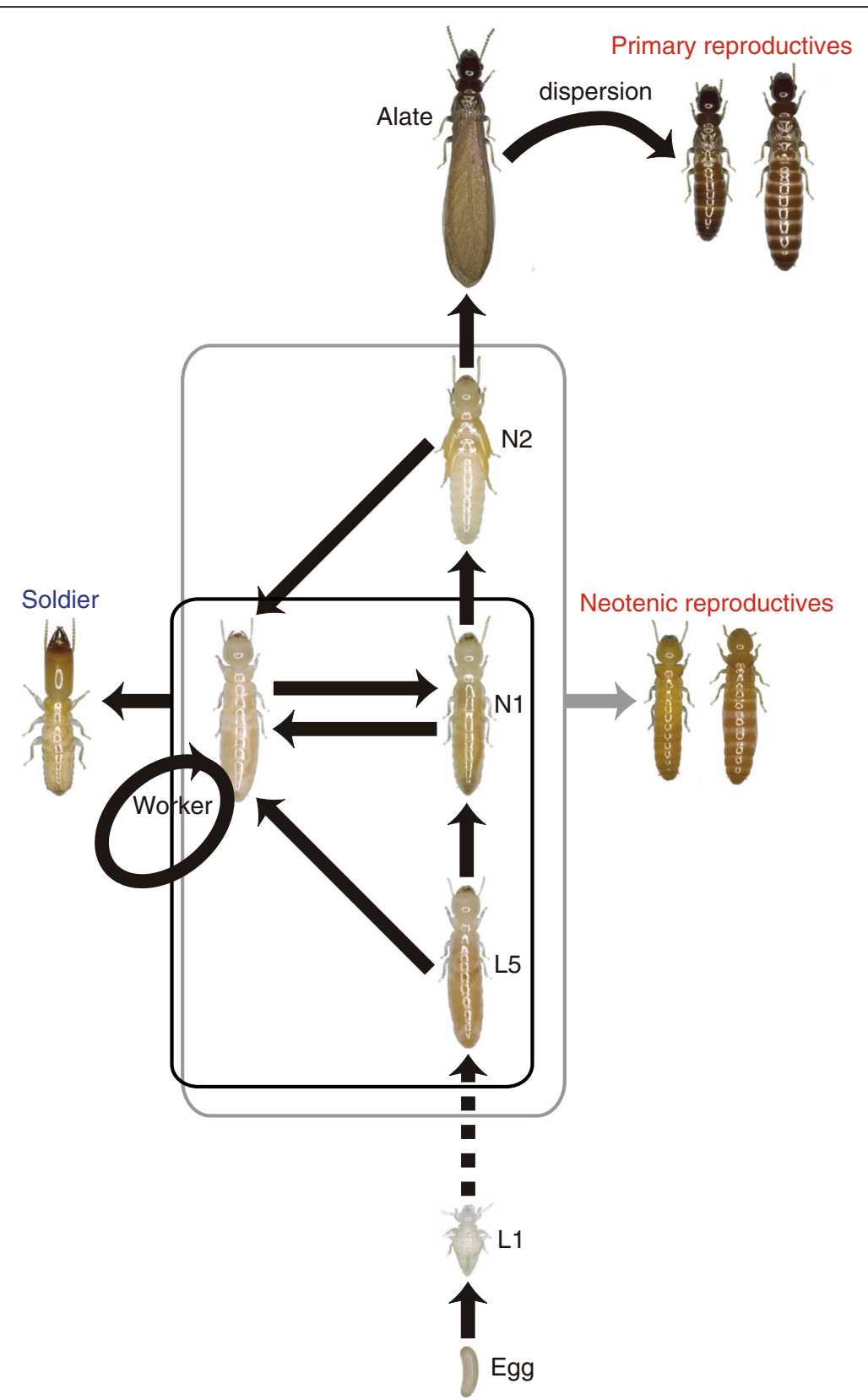

Fig. 1 The caste-developmental pathway in Kalotermitidae. L1-L5, first- to fifth-instar larva; N1-N2, first- to second-instar nymph

We also compared the hatching success of unfertilized eggs between all-female and mixed-sex populations. As expected, the all-female populations exhibited high rates of hatching success of unfertilized eggs (112 of 134 eggs [83.6\%] hatched) (Fig. 3). Notably, some unfertilized eggs from the mixed-sex population also hatched (7 of 193 eggs [3.6\%] hatched), indicating the presence of tychoparthenogenesis (occasional development of eggs without fertilization) $[1,28,29]$ in sexual colonies of this species (Fig. 3). No significant difference in hatching success was observed between unfertilized eggs of the all-female population (112 of 134 eggs [83.6\%] hatched) and fertilized eggs of the mixed-sex population (117 of 127 eggs [92.1\%] hatched) ( $P=0.12$, Fisher's exact probability test with Bonferroni correction) (Fig. 3). Overall, our results clearly indicate complete asexuality in the Shikoku and Kyushu populations, in contrast to the other normal sexual populations (Fig. 2b).

\section{A single origin of asexuality in G. nakajimai}

To elucidate the evolutionary relationships among asexual and sexual populations of G. nakajimai, we 

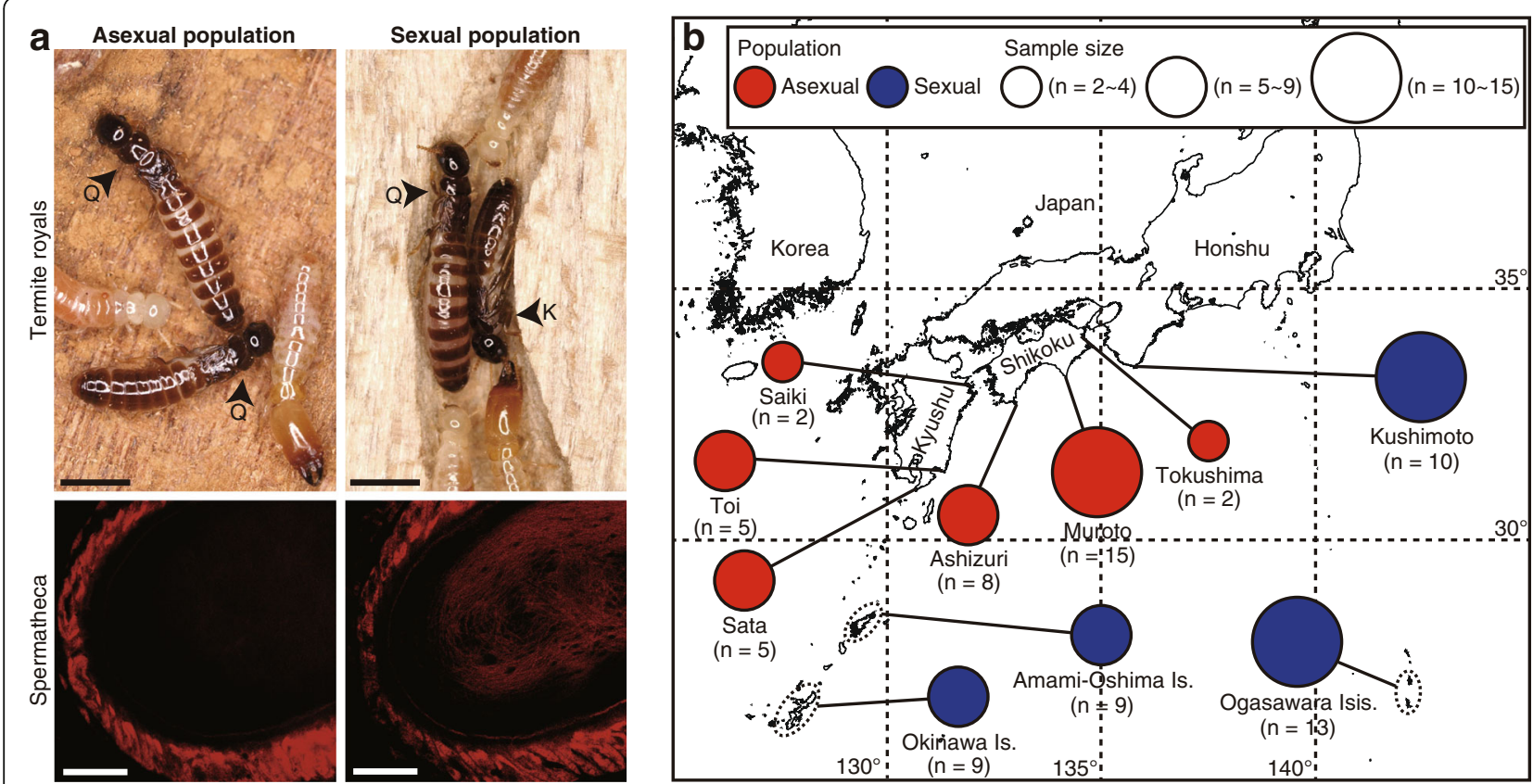

Fig. 2 Asexual and sexual populations of the termite Glyptotermes nakajimai. a Termite royals in mature colonies of asexual (top left) and sexual (top right) populations, and spermathecae of egg-laying queens without sperm in an asexual population (bottom left) and with sperm in a sexual population (bottom right). Spermathecae were stained by propidium iodide and observed under a confocal fluorescence microscope. Q, queen; K, king. Scale bars, 2 mm (top); 20 m (bottom). b Geographical distribution of asexual and sexual populations. Each population may include more than one collection site if they are located less than $50 \mathrm{~km}$ apart. The number of colonies sampled in each population is shown in parentheses

conducted phylogenetic analyses of Glyptotermes termites based on two independently evolving markers: mitochondrial cytochrome $c$ oxidase subunit II (COII) and nuclear internal transcribed spacer 2 (ITS2) sequences. The monophyly of G. nakajimai was unequivocally supported in each analysis (COII: Bayesian posterior probability $[\mathrm{BPP}]=1.00$; RAxML bootstrap support $[\mathrm{RBS}]=100 \%, \quad$ ITS2: $\mathrm{BPP}=1.00 ; \quad \mathrm{RBS}=100 \%$ ) (Fig. 4, Additional file 2: Figure S2). All sequences of COII and ITS2 from six collection sites across the range of asexual populations were identical (COII: 100\% identity, ITS2: 100\% identity). Likewise, COII and ITS2 sequences from nine collection sites across the range of sexual populations were highly similar to each other (COII: $99 \%-100 \%$ identity, ITS2: $96 \%-100 \%$ identity), and the monophyly of all sexual populations was well-supported (COII: BPP $=1.00$; RBS $=93 \%$, ITS2: BPP $=0.97 ; \mathrm{RBS}=100 \%)($ Fig. 4, Additional file 2: Figure S2). Given that all other known species of termites, including members of the genus Glyptotermes, are sexual, these results demonstrate a single origin of asexuality within $G$. nakajimai. The estimated divergence time between the two lineages was 14.1 million years ago (95\% confidence interval = 8.1-22.4 million years ago [Mya]) (Fig. 5). Whether asexuality has persisted in G. nakajimai for this period of time is not known. It is possible that the most closely related sexual relatives of the asexual G. nakajimai were not represented among our samples (perhaps because they are extinct, or found in areas we did not collect from), and that asexuality evolved much more recently than 14.1 Mya. Further work is required to investigate this issue.

Previous work has shown that the external morphology and cuticular hydrocarbon profiles of G. nakajimai representatives across its range are indistinguishable [21], including the asexual populations reported here. To further investigate potential fine-scale differences between the asexual and sexual lineages, we performed karyotyping. The sexual lineage consistently displayed $2 \mathrm{n}=34$ chromosomes (female: $n=12$, male: $n=12$ ), while the asexual lineage consistently harbored a complement of $2 n=35(n=18)$ and contained a trisomy, most probably of chromosome 16 (Fig. 6). To confirm our observations that the asexual lineage has an extra chromosome, we compared genome size (based on the $C$ value or nuclear DNA mass) between the asexual and sexual lineages. As expected, the genome size of the asexual lineage was significantly higher than that of the sexual lineage (colony: $F_{4,24}=0.80, P=0.54$; lineage: $F_{1}$, ${ }_{24}=55.76, \quad P<0.0001$; nested ANOVA with colonies nested within lineages [the asexual lineage and the sexual lineage]), although the ploidy levels were similar 
Table 1 Compositions of mature field colonies in asexual populations

\begin{tabular}{|c|c|c|c|c|c|c|}
\hline \multirow[b]{2}{*}{ Colony code ${ }^{a}$} & \multirow[b]{2}{*}{ Location } & \multicolumn{3}{|c|}{ No. reproductives $(f /[f+m])$} & \multirow{2}{*}{$\begin{array}{l}\text { No. } \\
\text { soldiers } \\
(\mathrm{f} /[\mathrm{f}+ \\
\mathrm{m}])\end{array}$} & \multirow{2}{*}{$\begin{array}{l}\text { No. } \\
\text { workers } \\
(f /[f+m])\end{array}$} \\
\hline & & A-derived & Neo. & Total & & \\
\hline & Shikoku, Japan & & & & & \\
\hline TO150911A & Tokushima, Tokushima & $6 / 6$ & $1 / 1$ & $7 / 7$ & $17 / 17$ & $100 / 100$ \\
\hline TO150911B & Tokushima, Tokushima & $2 / 2$ & $1 / 1$ & $3 / 3$ & $4 / 4$ & $100 / 100$ \\
\hline MR150216B & Muroto, Kochi & $1 / 1$ & $1 / 1$ & $2 / 2$ & $9 / 9$ & $100 / 100$ \\
\hline MR150216C & Muroto, Kochi & $1 / 1$ & $1 / 1$ & $2 / 2$ & $3 / 3$ & $100 / 100$ \\
\hline MR150216E & Muroto, Kochi & $2 / 2$ & $0 / 0$ & $2 / 2$ & $8 / 8$ & $100 / 100$ \\
\hline MR150216G & Muroto, Kochi & $5 / 5$ & 0/0 & $5 / 5$ & $14 / 14$ & $100 / 100$ \\
\hline MR150216H & Muroto, Kochi & $3 / 3$ & $1 / 1$ & $4 / 4$ & $11 / 11$ & $100 / 100$ \\
\hline MR150216l & Muroto, Kochi & $6 / 6$ & $0 / 0$ & $6 / 6$ & $15 / 15$ & $100 / 100$ \\
\hline MR150216」 & Muroto, Kochi & $5 / 5$ & $1 / 1$ & $6 / 6$ & $12 / 12$ & $100 / 100$ \\
\hline MR150216K & Muroto, Kochi & $3 / 3$ & $2 / 2$ & $5 / 5$ & $12 / 12$ & $100 / 100$ \\
\hline MR150216M & Muroto, Kochi & $2 / 2$ & 0/0 & $2 / 2$ & $8 / 8$ & 100/100 \\
\hline MR150217B & Muroto, Kochi & $5 / 5$ & $5 / 5$ & $10 / 10$ & $17 / 17$ & $100 / 100$ \\
\hline MR150217C & Muroto, Kochi & $2 / 2$ & $1 / 1$ & $3 / 3$ & $4 / 4$ & $100 / 100$ \\
\hline MR150217D & Muroto, Kochi & $2 / 2$ & $2 / 2$ & $4 / 4$ & $13 / 13$ & $100 / 100$ \\
\hline MR150910A & Muroto, Kochi & $12 / 12$ & $1 / 1$ & $13 / 13$ & $14 / 14$ & $100 / 100$ \\
\hline MR150910B & Muroto, Kochi & $7 / 7$ & $1 / 1$ & $8 / 8$ & $22 / 22$ & 100/100 \\
\hline MR150910D & Muroto, Kochi & $16 / 16$ & 0/0 & $16 / 16$ & $13 / 13$ & $100 / 100$ \\
\hline AS141110A & Ashizuri, Kochi & $10 / 10$ & $1 / 1$ & $11 / 11$ & $17 / 17$ & $100 / 100$ \\
\hline AS141110B & Ashizuri, Kochi & $5 / 5$ & 0/0 & $5 / 5$ & $1 / 1$ & $100 / 100$ \\
\hline AS141110D & Ashizuri, Kochi & $6 / 6$ & $3 / 3$ & $9 / 9$ & $6 / 6$ & $100 / 100$ \\
\hline AS141111A & Ashizuri, Kochi & $3 / 3$ & $2 / 2$ & $5 / 5$ & $1 / 1$ & $100 / 100$ \\
\hline AS141111H & Ashizuri, Kochi & $3 / 3$ & $0 / 0$ & $3 / 3$ & $6 / 6$ & $100 / 100$ \\
\hline AS141111I & Ashizuri, Kochi & $2 / 2$ & 0/0 & $2 / 2$ & $17 / 17$ & $100 / 100$ \\
\hline AS141111J & Ashizuri, Kochi & $2 / 2$ & 0/0 & $2 / 2$ & $1 / 1$ & $100 / 100$ \\
\hline \multirow[t]{2}{*}{ AS141111K } & Ashizuri, Kochi & $8 / 8$ & $2 / 2$ & $10 / 10$ & $16 / 16$ & $100 / 100$ \\
\hline & Kyushu, Japan & & & & & \\
\hline SK150715A & Saiki, Oita & $2 / 2$ & $3 / 3$ & $5 / 5$ & $3 / 3$ & $100 / 100$ \\
\hline SK150715B & Saiki, Oita & $2 / 2$ & 0/0 & $2 / 2$ & $1 / 1$ & $100 / 100$ \\
\hline TI150324A & Toi, Miyazaki & $3 / 3$ & $0 / 0$ & $3 / 3$ & $7 / 7$ & $100 / 100$ \\
\hline TI150324B & Toi, Miyazaki & $2 / 2$ & 0/0 & $2 / 2$ & $1 / 1$ & $100 / 100$ \\
\hline TI150324C & Toi, Miyazaki & 0/0 & $3 / 3$ & $3 / 3$ & $4 / 4$ & $100 / 100$ \\
\hline TI150324D & Toi, Miyazaki & $4 / 4$ & 0/0 & $4 / 4$ & $3 / 3$ & $100 / 100$ \\
\hline TI150728A & Toi, Miyazaki & $2 / 2$ & $1 / 1$ & $3 / 3$ & $7 / 7$ & $100 / 100$ \\
\hline ST150322C & Sata, Kagoshima & $2 / 2$ & 0/0 & $2 / 2$ & $7 / 7$ & $100 / 100$ \\
\hline ST150323L & Sata, Kagoshima & $2 / 2$ & 0/0 & $2 / 2$ & $3 / 3$ & $100 / 100$ \\
\hline ST160304A & Sata, Kagoshima & $4 / 4$ & 0/0 & $4 / 4$ & $4 / 4$ & $100 / 100$ \\
\hline ST160304B & Sata, Kagoshima & $15 / 15$ & 0/0 & $15 / 15$ & $13 / 13$ & $100 / 100$ \\
\hline ST160304C & Sata, Kagoshima & $4 / 4$ & 0/0 & $4 / 4$ & $2 / 2$ & $100 / 100$ \\
\hline Total & & $161 / 161$ & $33 / 33$ & 194/194 & $316 / 316$ & $3700 / 3700$ \\
\hline Mean (SEM) & & & & $100(0)$ & $100(0)$ & $100(0)$ \\
\hline
\end{tabular}

All collected reproductives, soldiers, and 100 workers from each colony were sexed. A-derived: alate-derived; Neo: neotenic; $\mathrm{f} /[\mathrm{f}+\mathrm{m}]$, females/[females $+\mathrm{males}]$ ${ }^{a}$ Numbers in colony codes indicate the dates when colonies were collected. For example, colony TO150911A was collected on 11 September 2015 
Table 2 Compositions of mature field colonies in sexual populations

\begin{tabular}{|c|c|c|c|c|c|c|}
\hline \multirow[b]{2}{*}{ Colony code ${ }^{a}$} & \multirow[b]{2}{*}{ Location } & \multicolumn{3}{|c|}{ No. reproductives $(f /[f+m])$} & \multirow{2}{*}{$\begin{array}{l}\text { No. } \\
\text { soldiers } \\
(f /[f+m])\end{array}$} & \multirow{2}{*}{$\begin{array}{l}\text { No. } \\
\text { workers } \\
(f /[f+m])\end{array}$} \\
\hline & & A-derived & Neo. & Total & & \\
\hline & Honshu, Japan & & & & & \\
\hline IZ150430A & Izumo, Kushimoto, Wakayama & $13 / 22$ & $0 / 1$ & $13 / 23$ & 2/11 & $53 / 100$ \\
\hline IZ150430B & Izumo, Kushimoto, Wakayama & $3 / 5$ & $0 / 1$ & $3 / 6$ & $2 / 7$ & $49 / 100$ \\
\hline IZ150430C & Izumo, Kushimoto, Wakayama & $1 / 2$ & $0 / 1$ & $1 / 3$ & $6 / 17$ & $62 / 100$ \\
\hline SN150430B & Misaki, Kushimoto, Wakayama & $31 / 53$ & $2 / 3$ & $33 / 56$ & $19 / 30$ & $58 / 100$ \\
\hline SN150430C & Misaki, Kushimoto, Wakayama & $36 / 68$ & $11 / 25$ & $47 / 93$ & $17 / 29$ & $54 / 100$ \\
\hline SN150430D & Misaki, Kushimoto, Wakayama & $5 / 10$ & $1 / 1$ & $6 / 11$ & $7 / 9$ & $52 / 100$ \\
\hline SN150430E & Misaki, Kushimoto, Wakayama & $3 / 6$ & $3 / 4$ & $6 / 10$ & $2 / 7$ & $68 / 100$ \\
\hline SN150430F & Misaki, Kushimoto, Wakayama & $3 / 6$ & $0 / 1$ & $3 / 7$ & $4 / 8$ & $47 / 100$ \\
\hline SN150430G & Misaki, Kushimoto, Wakayama & $13 / 25$ & $2 / 2$ & $15 / 27$ & $5 / 12$ & $50 / 100$ \\
\hline \multirow[t]{2}{*}{ SN150501A } & Misaki, Kushimoto, Wakayama & $5 / 8$ & $10 / 14$ & $15 / 22$ & $14 / 33$ & $49 / 100$ \\
\hline & Amami-Oshima Is., Japan & & & & & \\
\hline NZ150526A & Naze, Kagoshima & $3 / 9$ & $3 / 3$ & $6 / 12$ & $2 / 8$ & $44 / 100$ \\
\hline NZ150526C & Naze, Kagoshima & $1 / 2$ & $3 / 4$ & $4 / 6$ & $4 / 12$ & $60 / 100$ \\
\hline NZ150526F & Naze, Kagoshima & $8 / 19$ & $0 / 0$ & $8 / 19$ & $6 / 15$ & $55 / 100$ \\
\hline NK150527A & Setouchi, Kagoshima & $0 / 1$ & $3 / 4$ & $3 / 5$ & $3 / 16$ & $49 / 100$ \\
\hline \multirow[t]{2}{*}{ NK150527C } & Setouchi, Kagoshima & $3 / 6$ & $0 / 0$ & $3 / 6$ & $13 / 22$ & $53 / 100$ \\
\hline & Okinawa Is., Japan & & & & & \\
\hline HD150225A & Hedo, Okinawa & $3 / 8$ & $0 / 0$ & $3 / 8$ & $6 / 17$ & $41 / 100$ \\
\hline HD150225B & Hedo, Okinawa & $4 / 7$ & $7 / 14$ & $11 / 21$ & $5 / 37$ & $40 / 100$ \\
\hline HD150225C & Hedo, Okinawa & $1 / 2$ & $3 / 4$ & $4 / 6$ & $4 / 6$ & $43 / 100$ \\
\hline HD150225D & Hedo, Okinawa & $2 / 3$ & $1 / 1$ & $3 / 4$ & $3 / 8$ & $42 / 100$ \\
\hline HD160328B & Hedo, Okinawa & $2 / 4$ & $0 / 0$ & $2 / 4$ & $0 / 9$ & $48 / 100$ \\
\hline HD160328C & Hedo, Okinawa & $2 / 4$ & $0 / 0$ & $2 / 4$ & $5 / 15$ & $59 / 100$ \\
\hline NJ150226A & Nakijin, Okinawa & $0 / 1$ & $2 / 6$ & $2 / 7$ & $1 / 4$ & $27 / 100$ \\
\hline YJ150226A & Yagaijijima, Okinawa & $2 / 4$ & $0 / 0$ & $2 / 4$ & $6 / 11$ & $45 / 100$ \\
\hline \multirow[t]{2}{*}{ YJ150226B } & Yagajijima, Okinawa & $1 / 3$ & $0 / 0$ & $1 / 3$ & $2 / 7$ & $48 / 100$ \\
\hline & Ogasawara Isis., Japan & & & & & \\
\hline CC151014A & Chichijima, Tokyo & $22 / 45$ & $1 / 2$ & $23 / 47$ & $8 / 19$ & $52 / 100$ \\
\hline CC151014B & Chichijima, Tokyo & $1 / 2$ & $0 / 0$ & $1 / 2$ & $1 / 10$ & $51 / 100$ \\
\hline CC151014G & Chichijima, Tokyo & $0 / 1$ & $5 / 5$ & $5 / 6$ & $7 / 13$ & $42 / 100$ \\
\hline CC151014H & Chichijima, Tokyo & $3 / 5$ & $0 / 0$ & $3 / 5$ & $10 / 19$ & $43 / 100$ \\
\hline CC151015A & Chichijima, Tokyo & $1 / 3$ & $2 / 3$ & $3 / 6$ & $14 / 21$ & $43 / 100$ \\
\hline CC151015B & Chichijima, Tokyo & $7 / 14$ & $6 / 10$ & $13 / 24$ & $17 / 36$ & $38 / 100$ \\
\hline CC151015C & Chichijima, Tokyo & $2 / 4$ & $0 / 0$ & $2 / 4$ & $12 / 20$ & $53 / 100$ \\
\hline CC151015H & Chichijima, Tokyo & $1 / 3$ & $0 / 0$ & $1 / 3$ & $10 / 14$ & $53 / 100$ \\
\hline HH151016A & Hahajima, Tokyo & $0 / 0$ & $2 / 3$ & $2 / 3$ & $8 / 13$ & $47 / 100$ \\
\hline HH151016D & Hahajima, Tokyo & $6 / 10$ & $0 / 0$ & $6 / 10$ & $38 / 83$ & $56 / 100$ \\
\hline HH151016E & Hahajima, Tokyo & $12 / 23$ & $0 / 0$ & $12 / 23$ & $4 / 37$ & $24 / 100$ \\
\hline HH151016F & Hahajima, Tokyo & $11 / 25$ & $0 / 0$ & $11 / 25$ & $29 / 44$ & $46 / 100$ \\
\hline $\mathrm{HH} 151016 \mathrm{H}$ & Hahajima, Tokyo & $11 / 21$ & $1 / 2$ & $12 / 23$ & $12 / 34$ & $60 / 100$ \\
\hline Total & & $222 / 434$ & $68 / 114$ & $290 / 548$ & $308 / 713$ & $1804 / 3700$ \\
\hline Mean (SEM) & & & & $0.53(0.02)$ & $0.42(0.03)$ & $0.49(0.01)$ \\
\hline
\end{tabular}

All collected reproductives, soldiers, and 100 workers from each colony were sexed. A-derived, alate-derived; Neo., neotenic; f/[f + m], females/[females + males] ${ }^{a}$ Numbers in colony codes indicate the dates when colonies were collected. For example, colony IZ150430A was collected on 30 April 2015 


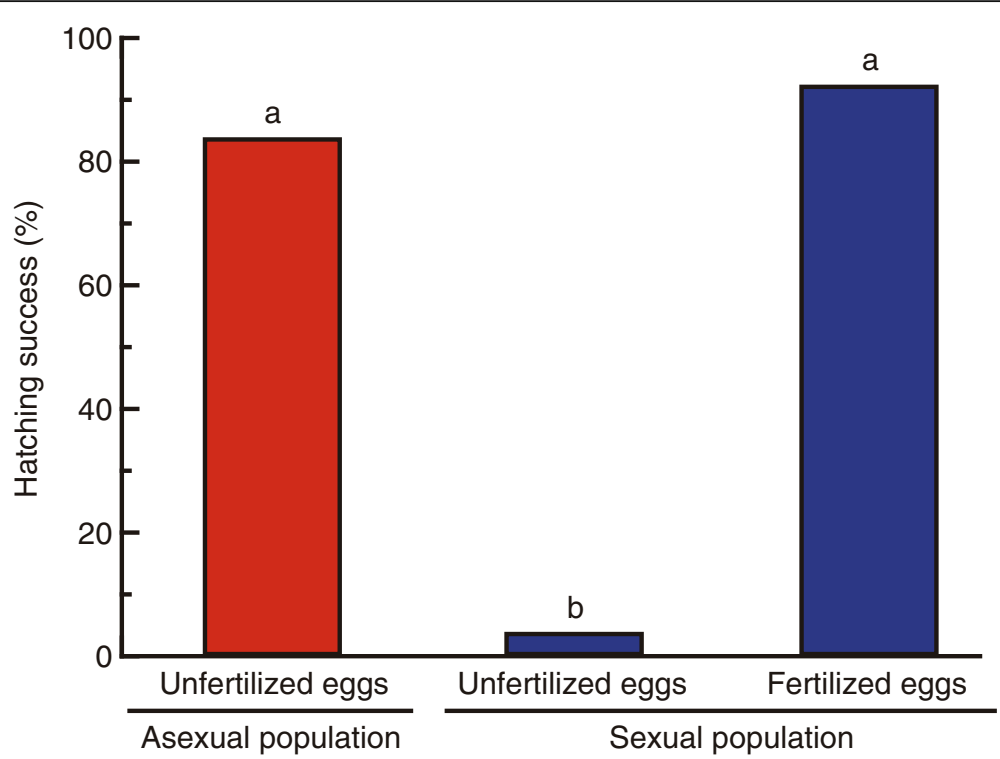

Fig. 3 Increased hatching success of unfertilized eggs in asexual populations. Comparison of the percentage of eggs hatched within 100 days after colony foundation among unfertilized eggs of an asexual population $(n=134)$, unfertilized eggs of a sexual population $(n=193)$, and fertilized eggs of a sexual population $(n=127)$. Different letters on the bars indicate significant differences $(P<0.0001$, Fisher's exact probability test with Bonferroni correction). For raw data, see Additional file 7

between the two lineages (Table 3). These results are consistent with the two lineages containing different chromosome profiles (Fig. 6), in addition to their molecular sequence profiles (Fig. 4, Additional file 2: Figure S2).

\section{Greater uniformity of head size in all-female soldiers of asexual colonies}

Phenotypic variations among individuals within a colony often provide opportunities for efficient task partitioning, such as sexual specialization in tasks in animal societies [13, 15, 30, 31]. However, such variations among individuals performing the same task may lower task-efficiency when individual variation reduces group performance. The most common defense mechanism in lower termites, including the genus Glyptotermes, is phragmosis, where soldiers with plug-like heads block tunnels connecting chambers, thus preventing enemies from invading the nest $[32,33]$. Tunnel width within a nest is relatively uniform, and stabilizing selection acts on soldier head width for efficient phragmotic defense in these termites, because soldiers with narrower head widths would tend to allow enemies to pass through more easily, while those with greater head width would tend to clog tunnels within the nest $[34,35]$. When sampling G. nakajimai in the field, we found that individuals of several ant species carried off struggling workers when logs infested with G. nakajimai were broken open. However, soldiers typically retreated to the small tunnels between chambers and plugged the tunnels with their heads to prevent the ants from invading intact parts of the nest (i.e., phragmotic defense) (Fig. 7a).

In the sexual lineage of G. nakajimai, male soldiers were significantly smaller than female soldiers in head width (colony: $F_{4,191}=5.16, P<0.001$, sex: $F_{1,191}=6.86$, $P<0.01$, two-way ANOVA), as well as in head length (colony: $F_{4,191}=10.67, P<0.0001$, sex: $F_{1,191}=13.21, P<$ 0.001, two-way ANOVA) (Additional file 3: Figure S3). Stabilizing selection appears to act on soldier head width because the head width to length ratio differed significantly between the sexes (colony: $F_{4,191}=12.46, P<$ 0.0001 , sex: $F_{1,191}=12.15, P<0.001$, two-way ANOVA), such that differences in head width between the sexes were reduced (Additional file 3: Figure S3).

To investigate whether asexuality leads to increased stabilization of soldier head width, we compared the within-colony coefficient of variation $(\mathrm{CV})$ of soldier head width between the asexual and sexual lineages. The asexual lineage had significantly smaller within-colony $\mathrm{CVs}$ of soldier head width than those of the sexual lineage ( $P=0.012$, Mann-Whitney $U$ test) (Fig. 7b), indicating reduced intracolonial variation in soldier head width in the asexual lineage. This is presumably due to a loss in sexual dimorphism in the asexual lineage, although the loss of genetic diversity resulting from parthenogenesis may also contribute. We also compared the proportion of soldiers to other individuals in mature field colonies between the asexual and sexual lineages. Mature field colonies of the asexual lineage had significantly lower proportions of soldiers to other individuals 


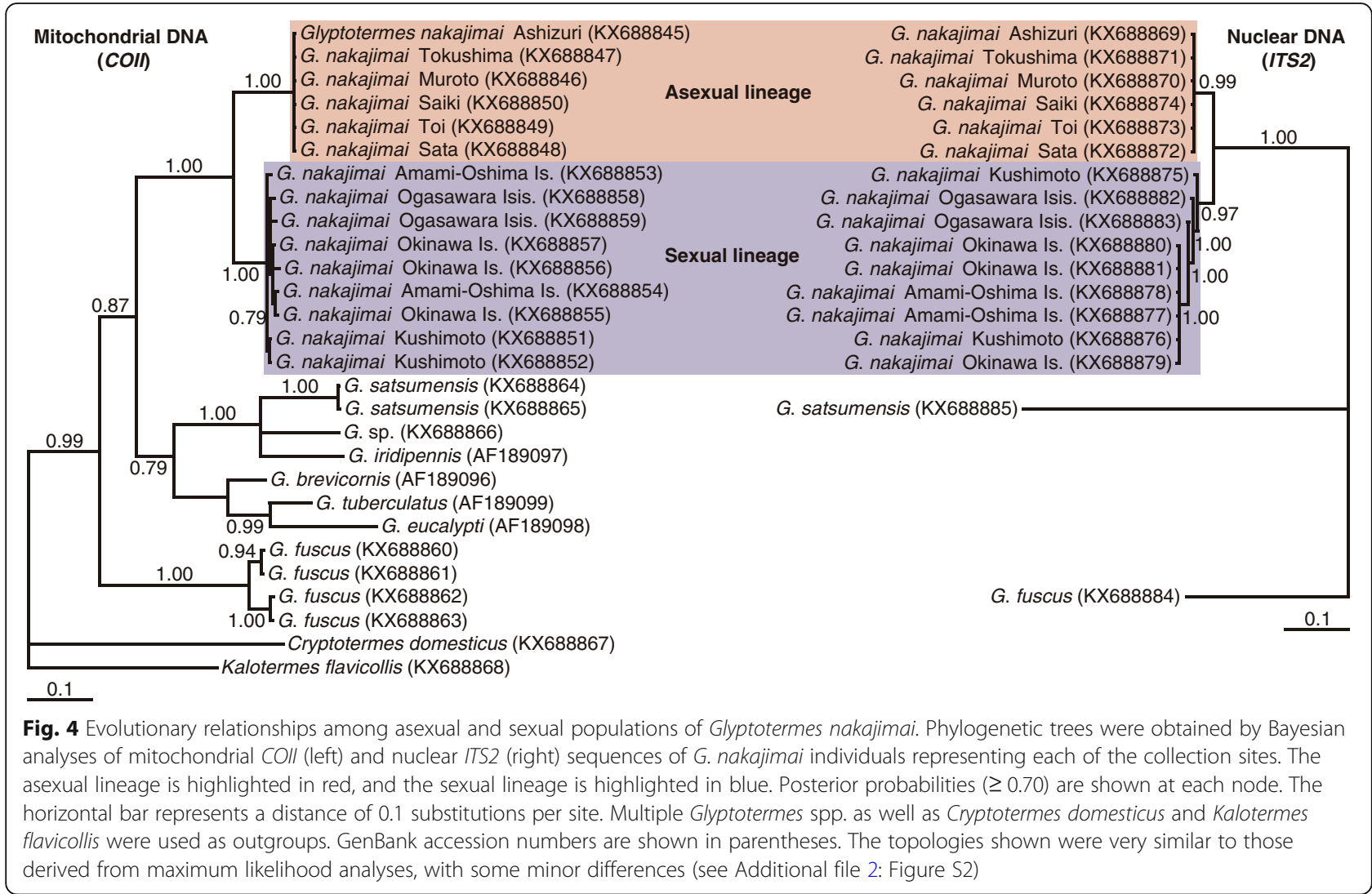

than those of the sexual lineage $(P<0.0001, \mathrm{GLM})$ (Fig. 7c), suggesting the number of soldiers required for colony defense in the asexual lineage appears to be lower than that in the sexual lineage. A reduction in the number of soldiers, which require sibling care because they are unable to feed themselves, is likely to allow investment elsewhere in the colony. Thus, our results hint at the possibility of increased defensive efficiencies arising from the greater morphological uniformity of soldiers in all-female asexual colonies. It is possible that such efficiencies contributed to the persistence and spread of the asexual lineage.

If the sexual lineage produced only female soldiers (or only male soldiers), intracolonial variation in soldier head width could also be reduced. Indeed, sexual specialization of soldiers is common in many termite taxa with sterile workers $[13,15]$. However, such sexual specialization is extremely rare in dry-wood termites (Kalotermitidae) [13, 15]. This can be partly explained by their caste-developmental pathway, through which all colony members finally develop into alates, neotenic reproductives (only a small proportion of individuals), or sterile soldiers [13,14] (Fig. 1). Sexual specialization of soldiers would therefore lead to skewed sex ratios of other colony members within colonies, most importantly alates. This would potentially lead to a failure of some alates from the colony to successfully breed.

\section{Pre-adaptive conditions for the evolution of maleless societies}

When the costs of asexuality are not high, a mutation causing asexual reproduction is expected to rapidly spread within a sexual population due to the twofold reproductive advantage of asexual reproduction. Under this scenario, asexual females produce only female offspring, while sexual females continue to produce both male and female offspring [36]. Assuming that approximately equal numbers of offspring are produced by both asexual and sexual colonies, asexual females will increase in frequency in the population and eventually replace sexual females entirely unless mutations that suppress asexuality arise.

We hypothesize the existence of a number of key traits in the ancestors of asexual G. nakajimai, which permitted them to overcome the barriers required for complete loss of males from their mixed-sex societies. Firstly, we hypothesize that the sexual ancestors of asexual G. nakajimai were likely to have been pre-adapted to overcome at least some developmental constraints associated with parthenogenesis. This is because parthenogenetic eggs have to be activated without sperm, and centrioles need to be inherited only from mothers during reproduction [5]. This hypothesis is supported by our finding of tychoparthenogenesis (occasional development of eggs 


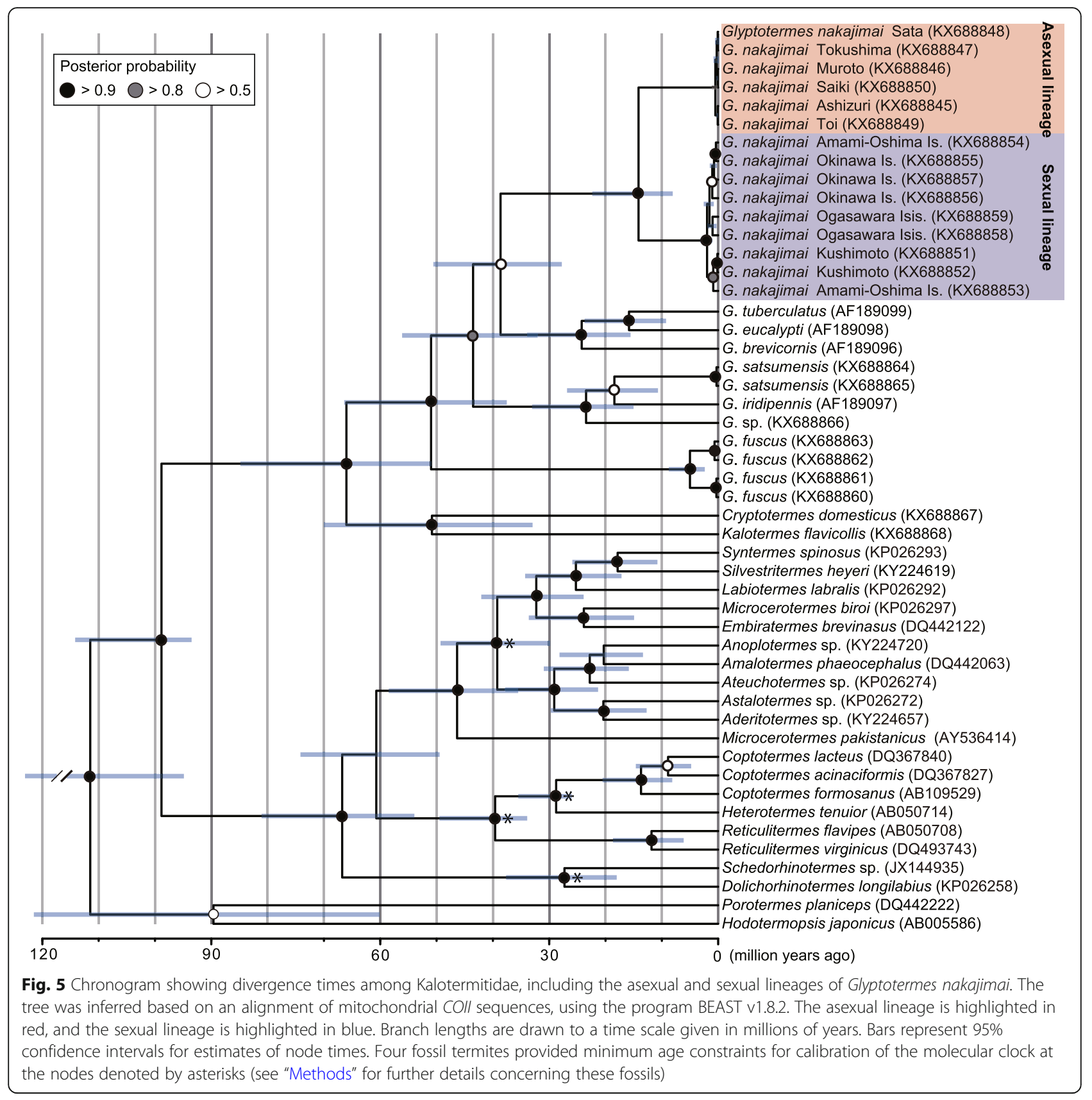

without fertilization) in the sexual lineage, whereby 7 of 193 unfertilized eggs (3.6\%) successfully developed into larvae (Fig. 3). Tychoparthenogenesis is thought to provide an important pathway to the evolution of parthenogenesis [29], but is also relatively common in many animal groups, regardless of the presence or absence of asexual lineages $[1,28]$. Therefore, tychoparthenogenesis is unlikely to be the only characteristic of the sexual ancestors of G. nakajimai that facilitated the evolution of all-female colonies.

The second trait we propose to have facilitated the transition to asexuality in G. nakajimai is cooperative colony foundation by queens (i.e., pleometrosis). Following the departure of alates from termite colonies on their nuptial flight, most termite incipient colonies are founded by a monogamous pair of primary (alate-derived) reproductives (i.e., a king and a queen) [37]. Both asexual and sexual G. nakajimai lineages produce alates that leave their colonies to undertake nuptial flights [21, 22]. However, rather than finding a single queen in incipient field colonies, we found that one incipient field colony of the asexual G. nakajimai lineage contained two queens, and three other incipient asexual field colonies were founded by more than two queens (range = 


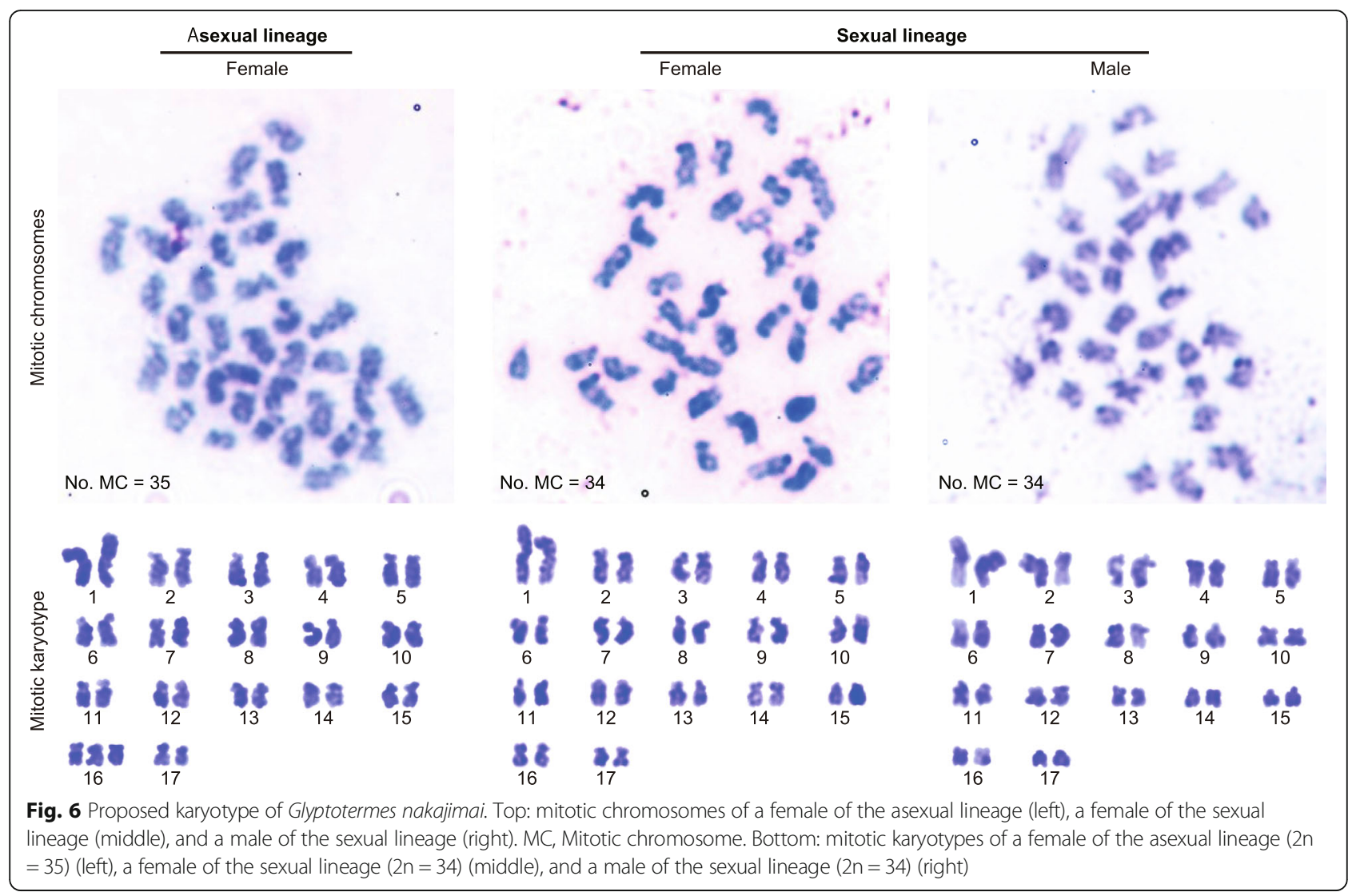

4-25). We found that the number of larvae per colony was positively correlated with the number of founders (Table 4). Incipient termite colonies typically experience high mortality rates, owing to disease and other factors $[38,39]$. Therefore, increased numbers of colony members through cooperative colony foundation by reproductives would be adaptive, especially in asexual populations, given the presumed genetic disadvantages of parthenogenesis [40]. In the present study, we were not able to find incipient field colonies of the sexual lineage of G. nakajimai. However, we found that 29 of 37 mature field colonies (78.4\%) of the sexual lineage, as well as 21 of 37 mature field colonies $(56.8 \%)$ of the asexual lineage, contained more than two alate-derived reproductives (Tables 1 and 2). This is suggestive of cooperative colony foundation by multiple kings and queens, although we cannot rule out the possibility of

Table 3 Comparison of genome size ( $C$ value) between the asexual and sexual lineages

\begin{tabular}{lll}
\hline Lineage & $C$-value $(\mathrm{pg})^{\mathrm{a}}$ & Relative ploidy level \\
\hline Asexual lineage & $1.27 \pm 0.00^{* * * *}$ & 2.07 \\
Sexual lineage & $1.23 \pm 0.00$ & 2 \\
\hline
\end{tabular}

Data for the asexual lineage was compared with the sexual lineage using nested ANOVA. ${ }^{* * * *}, P<0.0001$

avalues are mean $\pm \operatorname{SEM}(n=15)$ colony fusion. Based on these results, we hypothesize that the ancestors of the asexual lineage benefited from multi-queen colony foundation during the transition to male-free societies.

The presumed presence of multi-queen colony foundation in the ancestor of asexual G. nakajimai is also likely to have removed one potential barrier to the spread of the asexual phenotype: the expected failure of many colonies founded by single asexual queens [41]. Termites cannot clean their own bodies by self-grooming, with the exception of their antennae. Mutual grooming by reproductives during incipient colony foundation is therefore thought to play a key role in disease avoidance [41]. During the evolution of termite all-female asexual societies, females that founded colonies with other females (rather than alone) are likely to have been at a significant advantage, both from the benefits of grooming, as well as faster colony growth due to enhanced reproductive output.

The final traits we hypothesize that may have permitted the loss of males in G. nakajimai are those generally associated with the life histories of dry-wood termites. Dry-wood termites are single-site nesters, living in a single piece of dead wood that serves both as nest and as food [42]. This means that individual members do not need to forage outside the nest, reducing the risk of exposure to pathogens and parasites. Importantly, 


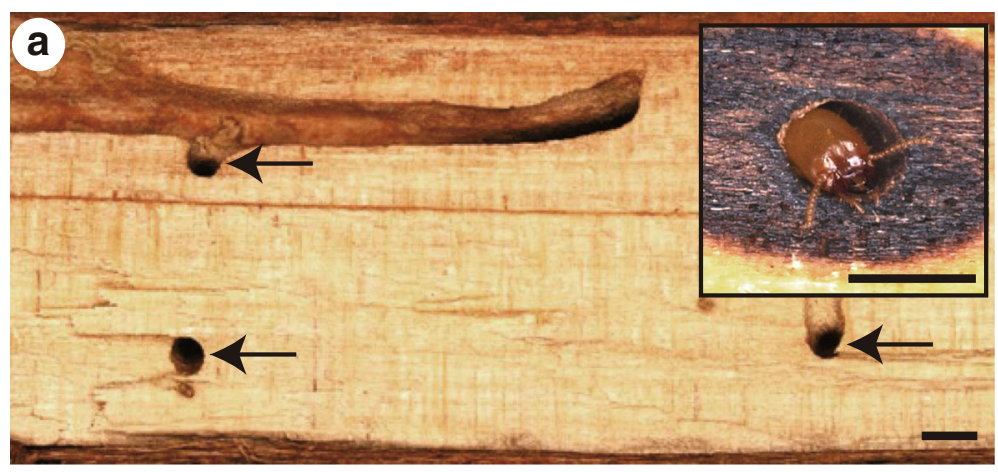

b


Fig. 7 Defensive advantage of colonies in the asexual lineage. a Cross section showing the structure of a Glyptotermes nakajimai nest. Small tunnels connecting chambers are indicated by arrows. (inset, right) A tunnel-blocking soldier (phragmotic defense). Scale bars, 2 mm. b Comparison of the within-colony coefficient of variation (CV) of soldier head width between the asexual and sexual lineages. Values are mean \pm SEM $(n=5)$. Individual data points are represented by open circles. ${ }^{*}, P<0.05$ (Mann-Whitney $U$ test). For raw data, see Additional file 7 . c

Comparison of the proportion of soldiers to other individuals of mature field colonies between the asexual and sexual lineages. Values are mean \pm SEM $(n=37)$. Individual data points are represented by open circles. ${ }^{* * *}, P<0.0001(G L M)$. For raw data, see Additional file 7 
Table 4 Compositions of incipient field colonies of the asexual lineage

\begin{tabular}{llll}
\hline Colony code $^{\mathrm{a}}$ & Location & No. primary queens & No. larvae \\
\hline MR150217A & Muroto, Kochi, Shikoku & 25 & 51 \\
AS141111B & Ashizuri, Kochi, Shikoku & 2 & 8 \\
AS141111D & Ashizuri, Kochi, Shikoku & 6 & 17 \\
ST150323F & Sata, Kagoshima, Kyushu & 4 & 12 \\
Mean (SEM) & & $9.25(5.31)$ & $22.00(9.84)$ \\
\hline
\end{tabular}

${ }^{a}$ Numbers in colony codes indicate the dates when colonies were collected. For example, colony MR150217A was collected on 17 February 2015

dry-wood nesters can easily disperse over water through wood-rafting. This is evidenced by the presence of many species, including G. nakajimai, in coastal areas and on remote islands $[20,21,43,44]$. According to the Red Queen hypothesis, sexual reproduction is favored because it helps the population to co-evolve with specialist parasites and pathogens [45]. Long-distance dispersal events might have released sexually reproducing ancestors of asexual G. nakajimai from selection pressures favoring sexual reproduction. This is because expansion into new areas may allow founders to escape from parasites and pathogens [46, 47]. Moreover, following dispersal to new habitats, asexuality could be adaptive because it is an effective way of circumventing the challenges associated with low population densities, such as inbreeding depression and the inability to find mates [48]. Indeed, many asexual lineages of ants are unusually widespread geographically [9].

\section{The mode of parthenogenesis in G. nakajimai}

The transition from sexual to parthenogenetic reproduction may have significant negative consequences as a result of increased homozygosity and inbreeding depression [5]. Such increases in homozygosity may occur under two forms of automictic parthenogenesis: gamete duplication or terminal fusion of gametes produced during meiosis. On the other hand, heterozygosity may be maintained in offspring under alternative forms of automictic parthenogenesis: central fusion of gametes produced during meiosis, or apomixis (in which eggs cells are essentially clones produced via mitosis) [5]. Indeed, the mode of thelytokous parthenogenesis in asexual lineages of hymenopteran social insects is either automixis with central fusion or apomixis $[8,9]$. The presence of an extra chromosome in all examined members of the G. nakajimai asexual lineage $(2 \mathrm{n}=35$, vs $2 \mathrm{n}$ $=34$ in members of the sexual lineage; Fig. 6 ) is suggestive of apomixis, as opposed to automixis. This is because an uneven number of chromosomes is likely to lead to pairing problems during the first stage of meiosis. Apomixis, on the other hand, would lead to a consistent number of chromosomes being passed to offspring [5]. We performed a preliminary investigation of the mode of parthenogenesis in G. nakajimai by genotyping individuals from both the asexual and sexual lineages (including offspring produced through tychoparthenogenesis from the sexual lineage; Fig. 3) at six polymorphic microsatellite markers developed for Glyptotermes termites (Additional file 4: Table S1). In the sexual lineage of G. nakajimai, offspring produced by tychoparthenogenesis were homozygous for a single maternal allele at two polymorphic loci (Gly08 and Gly18; Additional file 5: Table S2) (Additional file 6: Table S3). This pattern is suggestive of either (a) automixis with terminal fusion, where offspring are homozygous for a single maternal allele at all loci if no crossing-over takes place, as reported in other lower termites [16, 49], or (b) automixis with gamete duplication where offspring are completely homozygous for a single maternal allele at all loci, as suggested in a study of higher termites [16]. Unfortunately, the six markers we analyzed showed no polymorphism in the asexual lineage of G. nakajimai (Additional file 5: Table S2). Therefore, further work involving deeper sampling of the genomes of G. nakajimai representatives is required to test the hypothesis that the mode of parthenogenesis in the asexual lineage is apomixis, as suggested by its uneven number of chromosomes (Fig. 6). If our hypothesis is correct, such sampling of the asexual and sexual lineages could also determine whether heterozygous genotypes in the asexual lineage (if present) are derived from the conservation of initial allelic diversity present in the sexual ancestor. Under this scenario, the asexual lineage would have evolved through a transition from normal sexual reproduction directly to apomixis, as is known in Timema stick insects [6]. Alternatively, any heterozygous genotypes in the asexual lineage could be derived from new mutations, indicating that the asexual lineage evolved through an automictic step (as appears to be the case in tychoparthenogenetic G. nakajimai (Additional file 6: Table S3)).

\section{Conclusion}

Asexual lineages of advanced social animals were previously recognized only among Hymenoptera, whose societies are essentially all-female $[8,9]$. In contrast to hymenopteran societies, the common presence in termite societies of both male and female workers and 
soldiers [12] indicates that a mixed-sex workforce is a key component of their life history. We have conclusively demonstrated the completely asexual nature of some $G$. nakajimai populations (Fig. 2a, b, Tables 1 and 2), and have shown that this evolved on a single occasion from sexual ancestors with mixed-sex colonies (Fig. 4, Additional file 2: Figure S2). Our findings show that, at least under certain ecological conditions, males are not essential for the maintenance of advanced animal societies in which they previously played an active social role.

We have shown that asexuality acts as a stabilizer of soldier head size, which we hypothesize is beneficial for efficient phragmotic defense in termites (Fig. 7b, c). We also propose that a combination of traits present in the ancestors of the asexual lineage, including tychoparthenogenesis (Fig. 3), colony foundation by multiple queens (Table 4), and a single-site nesting life history, facilitated the complete loss of males from mixed-sex societies. These traits presumably allowed a mutation causing asexuality to spread rapidly to fixation following its appearance in ancestral sexual populations, as expected due to the twofold reproductive advantage of asexual reproduction [36]. Although rigorous studies of these traits in termites are scarce, they might also apply to other termite species, especially in members of Glyptotermes. The presence of several primary reproductives within a colony, which is unusual for termites, seems to be relatively common in this genus (e.g., [50]). One reason why asexual termite lineages have not previously been discovered may be a lack of attention given to the sex of individual colony members, including workers and soldiers, which in termites are often collectively called "neuter castes" regardless of sex. The discovery of additional asexual lineages of termites in nature will be aided by morphological sexing of both reproductives and non-reproductives. Development of sex-linked genetic markers in many genera may allow for more convenient detection of asexuality in termites.

\section{Methods}

\section{Termite collection}

We collected 74 mature colonies and four incipient colonies, which comprise only primary reproductives and young larvae, of the dry-wood termite G. nakajimai from 15 sites across ten populations (Honshu [Kushimoto], Shikoku [Tokushima, Muroto, and Ashizuri], Kyushu [Saiki, Toi, and Sata], Amami-Oshima Island, Okinawa Island, and Ogasawara Islands, Japan) from November 2014 to March 2016 (Tables 1, 2, and 4). We very carefully dismantled nest woods and extracted all colony members using an aspirator and forceps. The reproductives (kings and queens), soldiers, workers (also called pseudergates), nymphs, alates, and young instars (see Fig. 1) from each colony were placed in a moist unwoven cloth in a 90-mm Petri dish and preserved at $25{ }^{\circ} \mathrm{C}$ until further use. Portions of workers and nymphs from each colony were kept in the laboratory as stock colonies in $90-\mathrm{mm}$ Petri dishes that contained damp chips of sliced Oregon pine wood at $25{ }^{\circ} \mathrm{C}$ under constant darkness until subsequent experiments. The sex of all collected reproductives, soldiers, and 100 workers from each colony was determined based on the configuration of the caudal sternites [23, 24] under a stereomicroscope (SZX7; Olympus, Tokyo, Japan). To compare the proportion of soldiers to other individuals of mature field colonies between asexual and sexual populations, we used general linear modeling (GLM) (JMP 9; SAS Institute, Cary, NC).

\section{Sexing G. nakajimai reproductives, soldiers, and workers}

To test the accuracy of sexing individuals using external morphology in G. nakajimai, we used reproductives, soldiers, and workers of three mature field colonies of the sexual lineage (SN150430B, NZ150526F, and HH151016F in Table 2). Under a stereomicroscope (SZX7; Olympus, Tokyo, Japan), five putative male and five putative female reproductives, five putative male and five putative female soldiers, and ten putative male and ten putative female workers were randomly chosen from each colony using the configuration of the caudal sternites $[23,24]$. The true sex was then ascertained through inspection of the gonads [51].

\section{Spermatheca analysis}

To examine the insemination status of egg-laying queens, we developed a spermatheca staining method to observe inseminated sperm. Two egg-laying queens were randomly chosen from each of the six field colonies collected from asexual populations (TO150911A, TO150911B, MR150910A, MR150910B, SK150715A, and TI150728A in Table 1) and from sexual populations (IZ150430B, SN150430C, SN150430E, NZ150526C, NK150527C, and CC151015C in Table 2). Queens were dissected in phosphate-buffered saline (PBS), and their spermathecae were removed under a stereomicroscope (SZX7; Olympus). Whole spermathecae of individual queens were immersed in fixation solution (4\% paraformaldehyde) for $30 \mathrm{~min}$ at room temperature, washed with PBST (PBS with $0.1 \%$ Triton X-100) three times (5 min each), transferred into staining solution (propidium iodide [Dojindo, Kumamoto, Japan], final concentration: $2 \mathrm{ng} / \mathrm{ml}$ in PBST), and incubated for $20 \mathrm{~min}$ at room temperature. The spermathecae were washed with PBST for $10 \mathrm{~min}$, mounted on glass slides with mounting medium (Vectashield H-1000; Vector Laboratories, Burlingame, CA), and kept at $4{ }^{\circ} \mathrm{C}$ until analysis. To acquire fluorescent images of 
spermathecae, we photographed the stained spermathecae using a confocal laser scanning microscope (TCS-SP8; Leica, Wetzlar, Germany) equipped with a $100 \times / 1.4$ oil HC PL APO objective. The parameter settings of the microscope were as follows: excitation wavelength, $561 \mathrm{~nm}$; laser intensity, 20-40\%; gain, 20-50; and laser type, DPSS $20 \mathrm{~mW} 561 \mathrm{~nm}$. To compare the rate of insemination of egg-laying queens between asexual and sexual populations, we used Fisher's exact probability test (Statistica 10; StatSoft, Tulsa, OK).

\section{Comparison of hatching success of unfertilized eggs between the asexual and sexual lineages}

To investigate the difference in costs of parthenogenesis between the asexual and sexual lineages, we compared the hatching success of unfertilized eggs between the two lineages. Virgin alates were obtained from three stock colonies of the asexual lineage (AS141110A, AS141111I, and AS141111K in Table 1) and of the sexual lineage (IZ150430C, SN150430F, and SN150501A in Table 2), separated by sex before swarming began, and maintained in $90-\mathrm{mm}$ Petri dishes containing moist unwoven clothes until they shed their wings. Then, two females, or a female and a male were randomly chosen from each colony and placed in a $52 \times 76$ - $\mathrm{mm}$ glass cell that contained mixed sawdust bait blocks, as described in a previous study [41]. All treatments were replicated 20 times for each colony. The glass-cell colonies were kept at $25{ }^{\circ} \mathrm{C}$ under constant darkness for 100 days. We counted eggs and larvae by checking the glass-cell colonies every 3 days. The hatching success, calculated as percentage of eggs hatched within 100 days after colony foundation, was compared among eggs of glass-cell colonies founded by pairs of female reproductives (i.e., unfertilized eggs) of the asexual lineage, those of glass-cell colonies founded by pairs of female reproductives (i.e., unfertilized eggs) of the sexual lineage, and those of glass-cell colonies founded by pairs of female and male reproductives (i.e., fertilized eggs) of the sexual lineage using Fisher's exact probability tests with Bonferroni correction (Statistica 10; StatSoft). Because egg protection behavior by reproductives is indispensable for egg survival, data for the glass-cell colonies in which at least one reproductive died were excluded from the analysis. Moreover, because there were no significant differences between the natal colonies and between the glass-cell colonies within egg types (unfertilized eggs of the asexual lineage, unfertilized eggs of the sexual lineage, and fertilized eggs of the sexual lineage), respectively $(P>0.05$, Fisher's exact probability test with Bonferroni correction [Statistica 10; StatSoft]), we pooled the data for both the natal colonies and the glass-cell colonies of each egg type.

\section{Phylogenetic analyses}

To infer intraspecific relationships among asexual and sexual populations of $G$. nakajimai, we conducted phylogenetic analyses of Glyptotermes termites. One worker randomly chosen from each of the 15 G. nakajimai collection sites (six collection sites of asexual populations and nine collection sites of sexual populations) was used for phylogenetic analyses. The sequences of other kalotermitid species obtained in this study and from GenBank were also used in the analyses. Heads of individual termites were ground in Chelex-100 resin solution (Bio-Rad, Richmond, CA) and DNA was extracted and purified in accordance with standard Chelex-based protocols [52]. Individuals were sequenced for the mitochondrial cytochrome $c$ oxidase subunit II (COII) and the nuclear internal transcribed spacer 2 (ITS2) genes. Primer sequences, PCR conditions, and sequencing methods are detailed in previous reports (COII: [53]; ITS2: [54]). We obtained COII (624 bp) and ITS2 (444-bp) sequences. Consensus sequences were aligned using the ClustalX algorithm [55] from the BioEdit 7.0.4.1 sequence editor [56] and corrected manually. Bayesian inference was performed using MrBayes 3.1.2 [57] with the GTR + I + G model for COII and with the $\mathrm{HKY}+\mathrm{G}$ model for ITS2 selected by the hierarchical likelihood ratio test (hLRT) in MrModeltest 2.3 [58]. Kalotermes flavicollis (Fabricius) (GenBank accession number KX688868) and G. fuscus Oshima (GenBank accession number KX688884) were used as outgroups for the phylogenetic analyses of COII and ITS2, respectively. Methods of phylogenetic analyses are described in a previous report [53].

We also analyzed the sequence alignments using maximum likelihood in RAxML version 7.7.1 [59]. The GTRGAMMA model was selected for the combined datasets and 100 bootstrap replicates were performed. Kalotermes flavicollis (GenBank accession number KX688868) and G. fuscus (GenBank accession number KX688884) were used as outgroups for the phylogenetic analyses of COII and ITS2, respectively.

The COII and ITS2 gene sequences obtained in this study were deposited in the DDBJ/EMBL/GenBank nucleotide sequence databases under accession numbers KX688845-KX688885.

\section{Divergence-dating analysis}

We analyzed the COII DNA sequence alignment with a relaxed molecular-clock model using the Bayesian phylogenetic software BEAST 1.8.2. Rate variation was modeled among branches using uncorrelated lognormal relaxed clocks [60], with a single model for all genes. A Yule speciation process was used for the tree prior [61], and posterior distributions of parameters, including the tree, were estimated using MCMC sampling. We 
performed two replicate MCMC runs, with the tree and parameter values sampled every 1000 steps over a total of 50 million generations. A maximum clade credibility tree was obtained using Tree Annotator within the BEAST software package with a burn-in of 10,000 trees. Acceptable sample sizes and convergence to the stationary distribution were checked using Tracer 1.5 [60]. The molecular clock was calibrated using four minimum age constraints based on the following fossils: Coptotermes priscus Emerson, $\sim 26$ million years old; Dolichorhinotermes dominicanus Schlemmermeyer and Cancello, 18 million years old; Anoplotermes sp., 18 million years old; and Reticulitermes antiquus (Germar), 44 million years old; (for further details, see [62]). Fossil calibrations were implemented as exponential priors on node times.

\section{Cytological analysis}

To examine the karyotypes of the asexual and sexual lineages, we used female alates of six stock colonies of the asexual lineage (TO150911B, MR150910B, AS141110A, SK150715A, TI150728A, and ST160304B in Table 1) and four stock colonies of the sexual lineage (IZ150430C, NK150527C, HD160328C, and HH151016F in Table 2), and male neotenic reproductives of two stock colonies of the sexual lineage (NK150527C and HH151016F in Table 2). Three alates or five neotenic reproductives were randomly chosen from each colony. The somatic chromosomes of alates and neotenic reproductives were observed using the lactic acid dissociation drying method described in previous studies $[41,63]$.

\section{Genome size analysis}

To investigate the difference in genome size between the asexual and sexual lineages, we used female workers of three mature field colonies of the asexual lineage (TO150911A, MR150910A, and ST160304B in Table 1) and of the sexual lineage (IZ150430A, NZ150526C, and HH151016D in Table 2). Using flow cytometry with propidium iodide staining, we estimated genome size $(C$ value). Tissue for flow cytometric analysis was processed with a Cycletest PLUS DNA Reagent Kit (Becton Dickinson, San Jose, CA). All procedures were performed in accordance with the manufacturer's supplied protocol. Five female workers were randomly chosen from each colony and their heads were ground together with the heads of female Drosophila melanogaster Meigen (strain Oregon $\mathrm{R})(1 \mathrm{C}=0.18 \mathrm{pg}[64])$, which served as an internal standard, with a tight-fitting pestle. Detailed descriptions of the nuclear isolation and staining are described in a previous report [65]. Stained nuclei were analyzed for DNA-PI fluorescence using an Accuri C6 Flow Cytometer (Becton Dickinson) at an excitation wavelength of $488 \mathrm{~nm}$. Approximately 1000 cells were acquired for each measurement, and gating was performed using Accuri C6 software v1.0.264.21 (Becton Dickinson). Each $C$ value (pg) was calculated based on the main peak of $2 \mathrm{C}$ cells $\left(\mathrm{G}_{0} / \mathrm{G}_{1}\right.$ phase) of $\mathrm{G}$. nakajimai and $D$. melanogaster. To compare the genome size $(C$ value) between the asexual and sexual lineages, we used nested ANOVA. Assuming that the sexual lineage is diploid, as are all known termite species [11], a relative ploidy level was assigned to the asexual lineage using the following formula: ploidy level = mean genome size of the asexual lineage/mean genome size of the sexual lineage $\times 2$.

\section{Examination of sex differences in soldier morphology}

To compare morphology between male and female soldiers, we used soldiers of five mature field colonies of the sexual lineage (SN150501A, CC151015B, HH151016D, HH151016F, and HH151016H in Table 2). Head width and head length were measured for all collected male and female soldiers, excluding severely damaged individuals, from each colony. Head width, head length, the head width to length ratio (head width/head length) were analyzed using two-way ANOVA (Statistica 10; StatSoft, Tulsa, OK).

\section{Comparison of the within-colony CV of soldier head width between the asexual and sexual lineages}

To investigate whether asexuality leads to stabilization of soldier head width, we compared the within-colony coefficient of variation $(\mathrm{CV}=$ standard deviation $/$ mean $)$ of soldier head width between the asexual and sexual lineages. Excluding severely damaged individuals, all collected soldiers from five mature field colonies of the asexual lineage (TO150911A, MR150217B, MR150910B, AS141110A, and AS141111I in Table 1) and of the sexual lineage (SN150501A, CC151015B, HH151016D, HH151016F, and HH151016H in Table 2) were used for comparison. Head width was measured under a stereoscope (SZX7; Olympus) using a digital imaging system (FLVFS-LS; Flovel, Tokyo, Japan). Within-colony CVs of soldier head width, adjusted for sample size differences [66], were compared between the asexual and sexual lineages using Mann-Whitney $U$ test (Statistica 10; StatSoft).

\section{Development of microsatellite markers}

To characterize the asexual and sexual lineages of $G$. nakajimai genetically, we developed six polymorphic microsatellite markers for termites of the genus Glyptotermes. Genomic DNA was extracted from a pool of ten individuals from a single colony of G. fuscus collected on Okinawa Island using the DNeasy tissue Kit (Qiagen, Hilden, Germany). Microsatellite enrichment was achieved using streptavidin-coated magnetic spheres 
according to the protocol described in a previous study [49]. Enrichment amplicons were cloned using a pGEM T-Easy cloning kit (Promega, Madison, WI) following the manufacturer's instructions. Inserted DNA obtained from the color-positive clones was amplified by PCR. PCR products were checked for the insert size by agarose gel electrophoresis with a 100-bp ladder, and PCR products exhibiting a single band of 400-1000 bp were sequenced. Primer sequences, PCR conditions, and sequencing methods are detailed in a previous report [49].

Primers were designed for the 18 inserts containing a microsatellite region with more than nine repeat units. Genomic DNA was extracted from each head of 15 individuals per colonies of G. fuscus collected on Okinawa Island, those of the sexual lineage of G. nakajimai (two to three colonies for each population: IZ150430A, IZ150430B, SN150430F, SN150501A, NZ150526A, NZ150526F, NK150527C, HD160328B, HD160328C, NJ150226A, YJ150226B, CC151014G, CC151015B, HH151016D, and HH151016E in Table 2) and those of the asexual lineage of G. nakajimai (three to four colonies for each population: TO150911A, TO150911B, MR150217B, MR150910B, MR150910D, AS141110A, AS141111H, AS141111K, SK150715A, SK150715B, TI150324A, TI150728A, ST160304A, ST160304B, and ST160304C in Table 1). PCR was carried out in accordance with the following protocol. Each of the $25 \mu \mathrm{L}$ reaction mixtures contained $0.5 \mu \mathrm{M}$ each primer (for detailed information on the primers, see Additional file 4: Table S1), $1 \times$ PCR buffer, $0.5 \times$ Q-solution, $0.5 \mathrm{mM} \mathrm{MgCl}_{2}, 0.25 \mathrm{mM}$ each dNTP, $0.75 \mathrm{U}$ Taq DNA polymerase (Qiagen), and $1 \mu \mathrm{L}$ of template DNA. The reactions were run according to the PCR cycle, which consisted of an initial denaturation step at $94{ }^{\circ} \mathrm{C}$ for $3 \mathrm{~min}$, followed by 35 cycles of $30 \mathrm{~s}$ at $94{ }^{\circ} \mathrm{C}$ and $60 \mathrm{~s}$ at $60{ }^{\circ} \mathrm{C}$, and one step at $72{ }^{\circ} \mathrm{C}$ for $2 \mathrm{~min}$ to complete the extension at the end. The PCR products were electrophoresed with the GeneScan-600 LIZ size standard (Applied Biosystems, Foster City, CA) on a 3500 Genetic Analyzer (Applied Biosystems).

Of 18 loci, polymorphisms within the Okinawa Island population of G. fuscus were found for six (Additional file 5: Table S2). Genetic diversity based on number of alleles, observed, and expected heterozygosities, and deviations from Hardy-Weinberg equilibrium for each locus in the Okinawa Island population of G. fuscus, the sexual and asexual lineages of G. nakajimai, respectively, were calculated using Genepop on the Web 4.2 [67]. When appropriate, Bonferroni correction for multiple tests (Statistica 10; StatSoft) was applied.

\section{Mode of parthenogenesis in G. nakajimai}

To assess the mode of parthenogenesis in the sexual lineage of G. nakajimai, we genotyped the primary queens and larvae in the glass-cell colonies founded by pairs of female reproductives (the asexual laboratory colonies) described above. All individuals used in this analysis were placed in vials containing $99.5 \%$ ( $\mathrm{vol} / \mathrm{vol}$ ) ethanol and stored until DNA extraction. Heads of queens or whole larvae were ground in Chelex-100 resin solution (Bio-Rad, Richmond, CA), and DNA was extracted and purified in accordance with standard Chelex-based protocols [52]. Individuals were genotyped at two polymorphic microsatellite loci: Gly8 and Gly18 (Additional file 4: Table S1 and Additional file 5: Table S2). PCR and electrophoresis were performed as described above.

\section{Additional files}

Additional file 1: Figure S1. Sexual dimorphism in the external morphology of Glyptotermes nakajimai. Ventral view (posterior-up) of the caudal sternites and dorsal view (anterior-up) of the gonads of male (left) and female (right) reproductives (top), soldiers (middle), and workers (bottom). 7th, seventh sternite; T, testes; OV, ovaries. Scale bars, $400 \mu \mathrm{m}$ (PDF $3880 \mathrm{~kb}$ )

Additional file 2: Figure S2. Maximum likelihood trees of mitochondrial COIl (left) and nuclear ITS2 (right) sequences of Glyptotermes nakajimai individuals representing each of the collection sites. The asexual lineage is highlighted in red, and the sexual lineage is highlighted in blue. Bootstrap values $>50 \%$ are shown by the branch. The horizontal bar represents a distance of 0.1 substitutions per site. GenBank accession numbers are shown in parentheses. (PDF $427 \mathrm{~kb}$ )

Additional file 3: Figure S3. Differences in the head width (left), the head length (middle), and the head width to length ratio (right) between female $(n=99)$ and male $(n=102)$ soldiers of the sexual lineage. Parameters of the box-and-whisker plots: line, median; box, first to third quartile; upper whisker, third quartile $+1.5 \times$ interquartile range; lower whisker $=$ first quartile $-1.5 \times$ interquartile range; black dots, outliers. ${ }^{*}, P$ $<0.01$; ${ }^{* *}, P<0.001$ (two-way ANOVA). For raw data, see Additional file 7. (PDF $274 \mathrm{~kb})$

Additional file 4: Table S1. Newly developed microsatellite markers for termites of the genus Glyptotermes. (DOC $40 \mathrm{~kb}$ )

Additional file 5: Table S2. Characterization of six microsatellite loci in the asexual, sexual lineages of Glyptotermes nakajimai, and the related species G. fuscus. (DOC 51 kb)

Additional file 6: Table S3. Genotypes of the primary queens (PQ) and larvae $(L)$ in the asexual laboratory colonies of the sexual lineage of Glyptotermes nakajimai. (DOC $45 \mathrm{~kb}$ )

Additional file 7: Supporting data. Raw data for Fig. 7b, c, Table 3, and Additional file 3: Figure S3. (XLSX $31 \mathrm{~kb}$ )

\section{Acknowledgements}

We thank Yasushi Miyaguni, Naoto Yoshioka, and Manabu Yashiro for research assistance and William O. H. Hughes, Shigeto Dobata, Yuki Mitaka, Simon Y. W. Ho, Thomas Bourguignon, Benjamin P. Oldroyd, Madeleine Beekman, Theodore A. Evans, Edward L. Vargo, and Laurent Keller for helpful discussion.

\section{Funding}

This work was partly supported by the Japanese Society for the Promotion of Science (JSPS) Kiban Kenkyu S Grant No. 25221206 (to K.M.) and a JSPS Postdoctoral Fellowship for Research Abroad No. 558 (to T.Y).

Availability of data and materials

DNA sequences are available from GenBank (accession numbers of the new sequences KX688845-KX688885). All other data generated or analyzed during this study are included in this published article and its supplementary information files. 


\section{Authors' contributions}

TY, NL, KK, and KM designed the research. TY, KK, TN, TF, NM, YN, and KM performed the research. TY, KK, TN, TF, and KM contributed the new analytic tools. TY, NL, and KK analyzed the data. TY and NL wrote the first draft of the manuscript, and all authors contributed to its completion. All authors read and approved the final manuscript.

\section{Ethics approval and consent to participate} Not applicable.

\section{Consent for publication}

Not applicable.

\section{Competing interests}

The authors declare that they have no competing interests.

\section{Publisher's Note}

Springer Nature remains neutral with regard to jurisdictional claims in published maps and institutional affiliations.

\section{Received: 18 February 2018 Accepted: 9 August 2018}

Published online: 25 September 2018

\section{References}

1. Bell G. The masterpiece of nature: the evolution and genetics of sexuality. San Francisco: University of California Press; 1982.

2. West SA, Lively CM, Read AF. A pluralist approach to sex and recombination. J Evol Biol. 1999:12:1003-12.

3. Burt A. Perspective: sex, recombination, and the efficacy of selection-was Weismann right? Evolution. 2000;54:337-51

4. Normark BB, Judson OP, Moran NA. Genomic signatures of ancient asexual lineages. Biol J Linn Soc. 2003;79:69-84.

5. Engelstädter J. Constraints on the evolution of asexual reproduction. BioEssays. 2008:30:1138-50.

6. Schwander T, Crespi BJ. Multiple direct transitions from sexual reproduction to apomictic parthenogenesis in Timema stick insects. Evolution. 2009;63: 84-103.

7. Oldroyd BP, Fewell JH. Genetic diversity promotes homeostasis in insect colonies. Trends Ecol Evol. 2007;22:408-13.

8. Wenseleers T, Van Oystaeyen A. Unusual modes of reproduction in social insects: shedding light on the evolutionary paradox of sex. BioEssays. 2011; 33:927-37.

9. Rabeling C, Kronauer DJC. Thelytokous parthenogenesis in eusocial hymenoptera. Annu Rev Entomol. 2013;58:273-92.

10. Ross L, Gardner A, Hardy N, West SA. Ecology, not the genetics of sex determination, determines who helps in eusocial populations. Curr Biol. 2013;23:2383-7.

11. Bergamaschi S, Dawes-Gromadzki TZ, Scali V, Marini M, Mantovani B. Karyology, mitochondrial DNA and the phylogeny of Australian termites. Chromosom Res. 2007;15:735-53.

12. Abe T, Bignell DE, Higashi M. Termites: evolution, sociality, symbioses, ecology. Dordrecht: Kluwer Academic Publishers; 2000.

13. Roisin Y. Diversity and evolution of caste patterns. In: Abe T, Bignell DE, Higashi M, editors. Termites: evolution, sociality, symbioses, ecology. Dordrecht: Kluwer Academic Publishers; 2000. p. 95-119.

14. Katoh H, Matsumoto T, Miura T. Alate differentiation and compound-eye development in the dry-wood termite Neotermes koshunensis (Isoptera, Kalotermitidae). Insect Soc. 2007:54:11-9.

15. Noirot C. Social structure in termite societies. Ethol Ecol Evol. 1989;1:1-17.

16. Matsuura K. Evolution of the asexual queen succession system and its underlying mechanisms in termites. J Exp Biol. 2017;220:63-72.

17. Fougeyrollas R, Křivánek J, Roy V, Dolejšová K, Frechault $S$, Roisin $Y$, et al. Asexual queen succession mediates an accelerated colony life cycle in the termite Silvestritermes minutus. Mol Ecol. 2017;26:3295-308.

18. Matsuura K, Nishida T. Comparison of colony foundation success between sexual pairs and female asexual pairs in the termite Reticulitermes speratus (Isoptera: Rhinotermitidae). Popul Ecol. 2001:43:119-24.

19. Kawatsu K, Matsuura K. Preadaptation for parthenogenetic colony foundation in subterranean termites Reticulitermes spp. (Isoptera: Rhinotermitidae). J Ethol. 2013;31:123-8.
20. Morimoto K. Glyptotermes nakajimai, a new termite from Japan (Isoptera: Kalotermitidae). Kontyû. 1973:41:470-4.

21. Takematsu Y, Yamaoka R. Taxonomy of Glyptotermes (Isoptera, Kalotermitidae) in Japan with reference to cuticular hydrocarbon analysis as chemotaxonomic characters. Esakia. 1997;37:1-14.

22. Nishiharu S, Sasaji H. Discovery of Glyptotermes nakajimai Morimoto (Isoptera) from Is. Aoshima, Fukui Pref., off the coast of the Japan Sea. Ent J Fukui. 1994;15:61-2. (in Japanese)

23. Zimet M, Stuart AM. Sexual dimorphism in the immature stages of the termite, Reticulitermes flavipes (Isoptera: Rhinotermitidae). Sociobiology. 1982;7:1-7.

24. Miyaguni Y, Sugio K, Tsuji K. Refinement of methods for sexing instars and caste members in Neotermes koshunensis (Isoptera, Kalotermitidae). Sociobiology. 2012;59:1217-22.

25. Afzal M, Salihah Z. Sex ratio, occurrence of parthenogenesis, ovarian development and oviposition behaviour of the primary reproductives of Bifiditermes beesoni (Gardner) (Isoptera, Kalotermitidae). J Appl Entomol. 1985;100:132-46.

26. Noirot C, Thorne BL. Ergatoid reproductives in Nasutitermes columbicus (Isoptera, Termitidae). J Morphol. 1988:195:83-93.

27. Yashiro T, Matsuura K. Termite queens close the sperm gates of eggs to switch from sexual to asexual reproduction. Proc Natl Acad Sci U S A. 2014: 111:17212-7.

28. Simon JC, Delmotte F, Rispe C, Crease T. Phylogenetic relationships between parthenogens and their sexual relatives: the possible routes to parthenogenesis in animals. Biol J Linn Soc. 2003;79:151-63.

29. Schwander T, Vuilleumier S, Dubman J, Crespi BJ. Positive feedback in the transition from sexual reproduction to parthenogenesis. Proc R Soc Lond B Biol Sci. 2010:277:1435-42

30. Bird R. Cooperation and conflict: the behavioral ecology of the sexual division of labor. Evol Anthropol. 1999:8:65-75.

31. Wilson EO. Sociobiology: the new synthesis (25th anniversary edition) Cambridge: Harvard University Press; 2000.

32. Krishna K. A generic revision and phylogenetic study of the family Kalotermitidae (Isoptera). Bull Am Mus Nat Hist. 1961;122:303-408.

33. Deligne J, Quennedey A, Blum MS. The enemies and defense mechanisms of termites. In: Hermann HR, editor. Social Insects Vol. 2. New York: Academic Press; 1981. p. 1-76.

34. Matsuura K. Colony-level stabilization of soldier head width for head-plug defense in the termite Reticulitermes speratus (Isoptera: Rhinotermitidae) Behav Ecol Sociobiol. 2002:51:172-9.

35. Matsuura K. A novel hypothesis for the origin of the sexual division of labor in termites: which sex should be soldiers? Evol Ecol. 2006;20:565-74.

36. Maynard Smith J. The origin and maintenance of sex. In: Williams GC, editor Group selection. Chicago: Aldine Atherton; 1971. p. 163-75.

37. Nalepa CA, Jones SC. Evolution of monogamy in termites. Biol Rev. 1991;66:83-97.

38. Lepage M, Darlington JPEC. Population dynamics of termites. In: Abe T, Bignell DE, Higashi M, editors. Termites: evolution, sociality, symbioses, ecology. Dordrecht: Kluwer Academic Publishers; 2000. p. 333-61.

39. Rosengaus RB, Traniello JFA, Bulmer MS. Ecology, behavior and evolution of disease resistance in termites. In: Bignell DE, Roisin Y, Lo N, editors. Biology of termites: a modern synthesis. Dordrecht: Springer; 2011. p. 165-91.

40. Hamilton WD. Sex versus non-sex versus parasite. Oikos. 1980;35:282-90.

41. Matsuura K, Fujimoto M, Goka K. Sexual and asexual colony foundation and the mechanism of facultative parthenogenesis in the termite Reticulitermes speratus (Isoptera, Rhinotermitidae). Insect Soc. 2004:51:325-32.

42. Abe T. Evolution of life types in termites. In: Kawano S, Connell JH, Hidaka T, editors. Evolution and coadaptation in biotic communities. Tokyo: University of Tokyo Press; 1987. p. 125-48.

43. Emerson AE, Schmidt KP. Geographical origins and dispersions of termite genera. Fieldiana Zool. 1955;37:465-521.

44. Scheffrahn RH, Křeček J, Chase JA, Maharajh B, Mangold JR. Taxonomy, biogeography, and notes on termites (Isoptera: Kalotermitidae, Rhinotermitidae, Termitidae) of the Bahamas and Turks and Caicos Islands. Ann Entomol Soc Am. 2006:99:463-86.

45. Lively CM. A review of red queen models for the persistence of obligate sexual reproduction. J Hered. 2010;101:S13-20.

46. Ladle RJ, Johnstone RA, Judson OP. Coevolutionary dynamics of sex in a metapopulation: escaping the red queen. Proc R Soc Lond B Biol Sci. 1993; 253:155-60.

47. Leung TLF, King KC, Wolinska J. Escape from the red queen: an overlooked scenario in coevolutionary studies. Oikos. 2012;121:641-5. 
48. Taylor CM, Hastings A. Allee effects in biological invasions. Ecol Lett. 2005;8: 895-908.

49. Kobayashi K, Miyaguni Y. Facultative parthenogenesis in the Ryukyu drywood termite Neotermes koshunensis. Sci Rep. 2016;6:30712.

50. Hill GF. Termites (Isoptera) from the Australian region. Melbourne: Council for Scientific and Industrial Research; 1942.

51. Thompson CB, Snyder TE. The 'third form,' the wingless reproductive type of termites: Reticulitermes and Prorhinotermes. J Morphol. 1920;34:590-633.

52. Yashiro T, Mitaka Y, Nozaki T, Matsuura K. Chemical and molecular identification of the invasive termite Zootermopsis nevadensis (Isoptera: Archotermopsidae) in Japan. Appl Entomol Zool. 2018;53:215-21.

53. Yashiro T, Matsuura K, Tanaka C. Genetic diversity of termite-egg mimicking fungi "termite balls" within the nests of termites. Insect Soc. 2011;58:57-64.

54. Maryańska-Nadachowska A, Drosopoulos S, Lachowska D, Kajtoch $七$, Kuznetsova VG. Molecular phylogeny of the Mediterranean species of Philaenus (Hemiptera: Auchenorrhyncha: Aphrophoridae) using mitochondrial and nuclear DNA sequences. Syst Entomol. 2010;35:318-28.

55. Thompson JD, Gibson TJ, Plewniak F, Jeanmougin F, Higgins DG. The CLUSTAL_X windows interface: flexible strategies for multiple sequence alignment aided by quality analysis tools. Nucleic Acids Res. 1997;25:4876-82.

56. Hall TA. BioEdit: a user-friendly biological sequence alignment editor and analysis program for Windows 95/98/NT. Nucleic Acids Symp Ser. 1999:41:95-8.

57. Ronquist F, Huelsenbeck JP. MrBayes 3: Bayesian phylogenetic inference under mixed models. Bioinformatics. 2003;19:1572-4.

58. Nylander JAA, Ronquist F, Huelsenbeck JP, Nieves-Aldrey JL. Bayesian phylogenetic analysis of combined data. Syst Biol. 2004:53:47-67.

59. Stamatakis A, Hoover P, Rougemont J. A rapid bootstrap algorithm for the RAxML web servers. Syst Biol. 2008:57:758-71.

60. Drummond AJ, Rambaut A. BEAST: Bayesian evolutionary analysis by sampling trees. BMC Evol Biol. 2007;7:214

61. Gernhard T. The conditioned reconstructed process. J Theor Biol. 2008;253:769-78.

62. Bourguignon T, Lo N, Šobotník J, Ho SYW, lqbal N, Coissac E, et al. Mitochondrial phylogenomics resolves the global spread of higher termites, ecosystem engineers of the tropics. Mol Biol Evol. 2017;34:589-97.

63. Matsuura K. A test of the haplodiploid analogy hypothesis in the termite Reticulitermes speratus (Isoptera: Rhinotermitidae). Ann Entomol Soc Am. 2002;95:646-9

64. Gregory TR, Johnston J. Genome size diversity in the family Drosophilidae Heredity. 2008:101:228-38.

65. Nozaki T, Matsuura K. Termite queens have disproportionately more DNA in their fat body cells: reproductive division of labor and endoreduplication. Entomol Sci. 2016;19:67-71.

66. Sokal RR, Rohlf FJ. The principles and practice of statistics in biological research. New York: Freeman; 1995.

67. Raymond M, Rousset F. GENEPOP (version 1.2): population genetics software for exact tests and ecumenicism. J Hered. 1995:86:248-9.

Ready to submit your research? Choose BMC and benefit from:

- fast, convenient online submission

- thorough peer review by experienced researchers in your field

- rapid publication on acceptance

- support for research data, including large and complex data types

- gold Open Access which fosters wider collaboration and increased citations

- maximum visibility for your research: over $100 \mathrm{M}$ website views per year

At $\mathrm{BMC}$, research is always in progress.

Learn more biomedcentral.com/submissions 